\title{
On Artin's L-functions of the algebraic curves uniformized by certain automorphic functions
}

\author{
By Shuji KONNO
}

(Received June 13, 1962)

(Revised Dec. 15, 1962)

\section{Introduction.}

Recently, S. S. Rangachari [4], considered Artin's L-functions as an analogy of Hasse's zeta-function, for the algebraic curves uniformized by modular functions belonging to congruence subgroups. He proved, in certain cases, that Artin's $L$-function can be expressed as a product of Dirichlet series, corresponding to cusp forms of degree 2, obtained by Hecke [3]. On the other hand G. Shimura [7] proved that Hasse's conjecture is also true for algebraic curves uniformized by automorphic functions belonging to an indefinite quaternion algebra. Argebraic curves of this type include the curves treated by Rangachari as special case. In this paper, we consider Artin's $L$-functions for those curves.

Our principal result is as follows. Let $\Phi$ be an indefinite quaternion algebra over the rational number field $\boldsymbol{Q}, \mathfrak{D}$ be a maximal order in $\Phi$. For a positive integer $N$ prime to the discriminant $d(\Phi)$ of $\Phi$, we denote by $\Gamma$ and $\Gamma_{N}$, respectively, the group of units in $\mathbb{D}$ with positive reduced norm and its subgroup consisting of elements such that $\gamma \equiv 1 \bmod N$. As $\Phi$ has a faithfull representation by real matrices of degree $2, \Gamma$ and $\Gamma_{N}$ are considered as Fuchsian groups on the upper half plane $\mathfrak{S}$. We can find an algebraic curve $\mathfrak{L}_{N}$ defined over $\boldsymbol{Q}$, whose function field is the field of automorphic functions with respect to the group $\Gamma_{N}$. Let $\rho_{1}$ be an absolutely irreducible representation of the group $\Gamma / \Gamma_{N}=G$ contained in the analytic representation of $G$ on the jacobian variety of $\Omega_{N}$. Considering $G$ as a subgroup of the group $\Omega$ $=G L(2, \boldsymbol{Z} / N \boldsymbol{Z}) /\{ \pm 1\}$, we denote by $\left\{\rho_{1}, \cdots, \rho_{r}\right\}$ the set of all inequivalent conjugate representations of $\rho_{1}$ relative to $\Omega$. Put $\operatorname{deg} \rho_{1}=m$ and $\chi=\chi_{1}+\cdots+\chi_{r}$ with $\chi_{i}=\operatorname{tr} \rho_{i}$. Then our main theorem asserts that the $m$-th power of Artin's $L$-function $L(\chi, s)^{m}$ defined for the curves can be expressed as a product of Dirichlet series obtained from the representation of modular correspondences by automorphic cusp forms of type $(\Gamma, \rho, 2)$ which are considered in [8]. Therefore, such a power $L(\chi, s)^{m}$ is meromorphic on the whole $s$-plane and satisfies a functional equation (Theorem 1). Further we prove that, if the 
above $\chi$ is a simple character of the group $\Omega$, then Artin's $L$-function $L(\chi, s)$ itself can be expressed by such Dirichlet series with functional equation (Theorem 2).

In $\S 1$, we recall some definitions and results on automorphic functions with respect to a discontinuous group obtained from an indefinite quaternion algebra (cf. [7]). The definition of Hecke operators in [7] and [8] are slightly different from each other, though they are of course essentially the same. The definition in [7] is given in connection with the congruence relation, while that of [8] is convenient for proving the functional equation. We study in $\S 2$, the relation between these two definitions of Hecke operators. In $\S 3$, we define the global Artin's $L$-function. Using [8, Theorem 1$]$ and the fact that the analytic representations of modular correspondences on the jacobian variety are equivalent to the representations in the vector space of cusp forms, we get our principal results.

Here I wish my hearty thanks to Professor G. Shimura for his kind and valuable suggestions.

Throughout the present paper we use the same notations and terminologies. as in [7].

\section{§ 1. Preliminaries.}

We denote by $\mathfrak{S}$ the complex upper half plane $\{z \in \boldsymbol{C} \mid \operatorname{Im}(z)>0\}$. For every $x=\left(\begin{array}{ll}a & b \\ c & d\end{array}\right) \in M_{2}(\boldsymbol{R})$ with $\operatorname{det}(x)>0$ and for every $z \in \mathfrak{S}$, we put

$$
x[z]=\frac{a z+b}{c z+d}, \quad j(x, z)=c z+d .
$$

Let $\Phi$ be an indefinite quaternion algebra over $\boldsymbol{Q}$ and $\mathfrak{o}$ be a maximal order in $\Phi$. As $\Phi \otimes_{Q} \boldsymbol{R}$ is isomorphic to $\boldsymbol{M}_{2}(\boldsymbol{R})$, we can fix an injection of $\Phi$ into $\boldsymbol{M}_{2}(\boldsymbol{R})$ and consider $\Phi$ as subring of $\boldsymbol{M}_{2}(\boldsymbol{R})$. If we denote by $N(\alpha)$ the reduced norm of an element $\alpha$ of $\Phi$, then $N(\alpha)$ is equal to the determinant of $\alpha$ considered as an element of $\boldsymbol{M}_{2}(\boldsymbol{R})$, therefore, if $N(\alpha)>0$ for an element $\alpha$ of $\Phi, \alpha[z]$ has a meaning. Denote by $\Gamma$ the group composed of all units in $\mathfrak{D}$ such that $N(\gamma)=1$. For any two sided $\mathfrak{D}$-ideal $\mathfrak{a}$, let $\Gamma_{\mathfrak{a}}$ be the subgroup of $\Gamma$ consisting of elements $\gamma$ satisfying $\gamma \equiv 1 \bmod a$. Then $\Gamma$ and $\Gamma_{\mathfrak{a}}$ are Fuchsian groups on $\mathfrak{S}$, if we consider them as subgroup of $\operatorname{SL}(2, \boldsymbol{R})$.

For every $z \in \mathscr{S}$, we denote by $\mathscr{L}(z)=\left(A_{z}, \mathcal{C}_{z}, \theta_{z}\right)$ the polarized abelian variety of type $\mathfrak{D}$ with the abelian variety $A_{z}$, the polarization $\mathcal{C}_{z}$, and the homomorphism $\theta_{z}$ of $\mathfrak{o}$ into $\mathcal{A}\left(A_{z}\right)$ considered in $[6,7]$. There exist a set of meromorphic functions $f_{1}(z), \cdots, f_{m}(z)$ on $\mathscr{S}$ and a discrete subset $\mathfrak{W}$ of $\mathfrak{S}$ such that the field of moduli of $\mathscr{Q}(z)$ are given by

$$
K_{1}=K_{1, z}=\boldsymbol{Q}\left(f_{1}(z), \cdots, f_{m}(z)\right) \quad \text { for every } \quad z \in \mathfrak{H}-\mathfrak{B},
$$


and the function field

$$
\mathscr{R}(\mathfrak{0})=\boldsymbol{C}\left(f_{1}, \cdots, f_{m}\right)
$$

is the field of automorphic functions on $\mathfrak{5}$ with respect to $\Gamma$.

Let $(V, h)$ be a normalized Kummer variety of $\mathcal{P}(z)$. We put

$$
\mathfrak{g}\left(\mathfrak{a}, A_{z}\right)=\left\{t \in A_{z} \mid \theta_{z}(\alpha) t=0 \text { for all } \alpha \in \mathfrak{a}\right\} \text {. }
$$

Denote by $K_{\mathfrak{a}}=K_{\mathfrak{a}, z}$ the field generated over $K_{1}$ by the points $h(t)$ for $t \in \mathfrak{g}\left(\mathfrak{a}, A_{z}\right)_{\text {. }}$ The extension $K_{\mathfrak{a}} / K_{1}$ does not depend on the choice of $(V, h)$ and is normal. Furthermore there exist meromorphic functions $g_{1}, \cdots, g_{M}$ on $\mathfrak{S}_{\text {g }}$ such that

$$
K_{\mathfrak{a}}=K_{a, z}=\boldsymbol{Q}\left(f_{1}(z), \cdots, f_{m}(z), g_{1}(z), \cdots, g_{M}(z)\right) \quad \text { for } \quad z \in \mathfrak{S}_{\mathfrak{g}}-\mathfrak{W}^{\prime},
$$

where $\mathfrak{W}^{\prime}$ is a certain countably infinite subset of $\mathfrak{S}$ containing $\mathfrak{W}$; and the function field

$$
\mathfrak{A}(\mathfrak{D} ; \mathfrak{a})=\boldsymbol{C}\left(f_{1}, \cdots, f_{m}, g_{1}, \cdots, g_{M}\right)
$$

is the field of automorphic functions on $\mathfrak{H}_{2}$ with respect to $\Gamma_{\mathbf{a}}$.

By Theorem 2 of [7], the galois group of $K_{\mathfrak{a}} / K_{1}$ is isomorphic to $G_{\mathfrak{a}} /\{ \pm 1\}$, where $G_{a}$ is the group of all regular elements in $\mathfrak{o} / \mathfrak{a}$. If $\mathfrak{a}$ is prime to the discriminant $d(\Phi)$ of $\Phi, \mathfrak{a}$ is written in the form $\mathfrak{a}=N_{\mathfrak{b}}$ for a positive integer $N$ prime to $d(\Phi)$; and $G_{a}$ is isomorphic to the group $G L(2, \boldsymbol{Z} / N \boldsymbol{Z})$ of all regular elements in $\boldsymbol{M}_{2}(\boldsymbol{Z} / N \boldsymbol{Z})$. Let $H$ be the subgroup of $G L(2, \boldsymbol{Z} / N \boldsymbol{Z})$ defined by

$$
H=\left\{\left(\begin{array}{rr}
a & 0 \\
0 & \pm 1
\end{array}\right) \mid a \in Z, \quad(a, N)=1\right\} .
$$

Denote by $L_{N}$ the subfield of $K_{\mathfrak{a}}$ corresponding to the subgroup $H$. Let $\mathfrak{Q}_{N}$ be an algebraic curve, complete and without singularities, having a generic point $u$ over $\boldsymbol{Q}$ such that $\boldsymbol{Q}(u)=L_{N}$, and $J_{N}$ be a jacobian variety of $\mathfrak{L}_{N}$, defined over $\boldsymbol{Q}$.

For every left $\mathrm{p}$-ideal $q$ with $N(q)=q$, let $X_{q}$ be the modular correspondence of $\Omega_{N}$ associated with $\mathfrak{q}$. For every integer $n$ prime to $N$, let $\tau_{n}$ be the automorphism of $K_{a}$ corresponding the element $n$ of $G_{a}$, and $Y_{n}$ be the locus of $u \times u^{\tau_{n}}$ over $\boldsymbol{Q}$ on $\mathfrak{\Sigma}_{N} \times \mathfrak{\Sigma}_{N}[\mathbf{5}, \S 4.3]$. Put $\psi=\left(\begin{array}{rr}0 & 1 \\ -1 & 0\end{array}\right)$. Denote by $Z$ the locus of $u \times u^{\psi}$ over $\boldsymbol{Q}\left(\zeta_{N}\right)$, when $\zeta_{N}=e^{2 \pi i / N}$. Let $\xi_{p}, \eta_{p}, \zeta$ be the endomorphism of $J_{N}$ determined by $X_{p}, Y_{p}, Z$ respectively.

When an algebraic geometric object $\boldsymbol{U}$ is defined over the field $k$ with a place $P$; we denote by $P(\boldsymbol{U})$ or $\tilde{\boldsymbol{U}}$ the reduction of $\boldsymbol{U} \bmod P$. Now $J_{N}$ is without defect for almost all $p$, and $\tilde{J}_{N}$ is a jacobian variety of $\widetilde{\mathfrak{L}}_{N}$; for such a $p$ we have the congruence relations

$$
\begin{aligned}
\tilde{\xi}_{p} & =\pi_{p}+\pi_{p}^{\prime} \circ \tilde{\eta}_{p} \\
\pi_{p}^{\prime} \circ \tilde{\eta}_{p} & =\tilde{\zeta}^{-1} \circ \pi_{p}^{\prime} \circ \tilde{\zeta}
\end{aligned}
$$


where $\pi_{p}$ denotes the $p$-th power endomorphism of $J_{N}$ and $\pi_{p}^{\prime}=p \cdot \pi_{p}^{-1}$.

By a representation of a group, we always understand a representation by complex matrices or in a complex vector space. The following lemma is well known [1].

LEMMA 1. Let $\Omega$ be a finite group and $G$ be a normal subgroup of $\Omega$. Then the following assertions hold.

i) If $\rho$ is an irreducible representation of $\Omega$, then the representation $\rho_{G}$ of $G$ obtained by restricting $\rho$ to $G$ is either itself irreducible or is fully reducible into irreducible components all of which are of the same degree and of the same multiplicities.

ii) If $\sigma$ is an irreducible component of $\rho_{G}$, then any other irreducible component $\sigma^{\prime}$ of $\rho_{G}$ is a conjugate of $\sigma$ relative to $\Omega$; namely there exists an element $\omega$ of $\Omega$ such that $\sigma^{\prime}(\gamma)=\sigma\left(\omega \gamma \omega^{-1}\right)$ for $\gamma \in G$; conversely, every $\sigma^{\prime}$ obtained from $\sigma$ in this manner is an irreducible component of $\rho_{G}$.

iii) Any irreducible representation of $G$ can be obtained as an irreducible component of $\Omega$.

\section{$\S 2$. Analytic representation of modular correspondences.}

Let $\mathfrak{a}=N_{\mathfrak{o}}(N$; positive integer $)$ be a two-sided $\mathfrak{D}$-ideal, such that $N$ is prime to the discriminant $d(\Phi)$ of $\Phi$. Denote by $\Delta_{N}$ the set of all elements $\alpha$ in $\mathfrak{D}$ such that $N(\alpha)$ is positive and prime to $N$. Hereafter we denote $\Gamma_{\mathfrak{a}}$ by $\Gamma_{N}$.

We call a complex valued holomorphic function $f(z)$ on $\mathfrak{S}$ a cusp form of degree 2 with respect to $\Gamma_{N}$, if

(i) $f(r[z])=j(\gamma, z)^{2} f(z) \quad$ for every $\quad r \in \Gamma_{N}$,

(ii) $f(z)$ vanishes at every cusp of $\Gamma_{N}$.

We denote by $S\left(\Gamma_{N}, 2\right)$ the set of all such cusp forms.

For every $\alpha \in \Delta_{N}$, let $\Gamma_{N} \alpha \Gamma_{N}=\bigcup_{i=1}^{m} \Gamma_{N} \alpha_{i}$ be a disjoint expression. We define the operators $\Gamma_{N} \alpha \Gamma_{N}\left(\alpha \in \Delta_{N}\right)$ on $S\left(\Gamma_{N}, 2\right)$ by

$$
f \mid\left(\Gamma_{N} \alpha \Gamma_{N}\right)(z)=N(\alpha) \sum_{i=1}^{m} f\left(\alpha_{i}[z]\right) j\left(\alpha_{i}, z\right)^{-2} .
$$

If $\gamma \in \Gamma$ and $f(z) \in S\left(\Gamma_{N}, 2\right), f(z) \rightarrow f(\gamma[z]) j(\gamma, z)^{-2}$ gives an automorphism of $S\left(\Gamma_{N}, 2\right)$. We obtain thus a representation of $\Gamma$ in $S\left(\Gamma_{N}, 2\right)$ and this representation induces an analytic representation of the group $G=\Gamma / \Gamma_{N}$ on the jacobian variety $J_{N}$. In the expression $\Gamma_{N} \alpha \Gamma_{N}=\bigcup_{i=1}^{m} \Gamma_{N} \alpha_{i}$ we can choose $\alpha_{i}$ so that $\alpha_{i} \equiv\left(\begin{array}{ll}1 & 0 \\ 0 & c\end{array}\right) \bmod N(\mathrm{cf} .[7]$ ). Using this fact, we can easily prove the following:

Proposition 1. Let $\alpha$ be an element in $\Delta_{N}$ with $N(\alpha) \equiv q \bmod N$. Then, for every $\gamma \in \Gamma$, we have

$$
\left(\Gamma_{N} \alpha \Gamma_{N}\right) \circ\left(\Gamma_{N} \gamma \Gamma_{N}\right)=\left(\Gamma_{N} \sigma_{q} \gamma \sigma_{q}^{-1} \Gamma_{N}\right) \circ\left(\Gamma_{N} \alpha \Gamma_{N}\right)
$$


where $\circ$ means the product as operator and $\sigma_{q}$ denotes an element of $\mathfrak{D}$ such that $\sigma_{q} \equiv\left(\begin{array}{ll}1 & 0 \\ 0 & q\end{array}\right) \bmod N$.

Besides the group $G=\Gamma / \Gamma_{N}$, we consider the group $\Omega=G L(2 ; \boldsymbol{Z} / N \boldsymbol{Z}) /\{ \pm 1\}$. Since $G$ is isomorphic to $S L(2, \boldsymbol{Z} / N \boldsymbol{Z}) /\{ \pm 1\}$, we may consider $G$ as a normal subgroup of $\Omega$. By Lemma 1 , for any absolutely irreducible representation. $\rho_{1}$ of $G$ contained in the analytic representation of $G$ on $J_{N}$, there exists an irreducible representation $\rho$ of $\Omega$ such that $\rho$ is equivalent to the direct. sum of $\rho_{1}, \cdots, \rho_{r}$ with the same multiplicity $\kappa$ :

$$
\rho \sim \kappa\left(\rho_{1}+\cdots+\rho_{r}\right)
$$

where $\rho_{1}, \cdots, \rho_{r}$ are inequivalent representation of $G$ which are conjugates of $\rho_{1}$ with respect to $\Omega$ (in the sense of Lemma 1 , ii)). With this irreducible representation $\rho$ of $\Omega$, we shall consider the linear space defined by

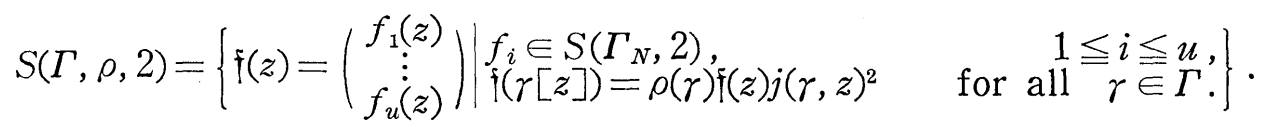

These vector functions $f(z)$ are nothing but the automorphic cusp forms of type $(\Gamma, \rho, 2)$ treated in [8]. Therefore we can define the operators $\Gamma \alpha \Gamma$ $\left(\alpha \in \Delta_{N}\right)$ on this space after [8]. Namely, when $\Gamma \alpha \Gamma=\bigcup_{i=1}^{m} \Gamma \alpha_{i}\left(\alpha \in \Delta_{N}\right)$ is a. disjoint sum we define

$$
\mathfrak{f} \mid(\Gamma \alpha \Gamma)(z)=\sum_{i=1}^{m} \rho\left(\alpha_{i}\right)^{-1} \mathfrak{f}\left(\alpha_{i}[z]\right) j\left(\alpha_{i}, z\right)^{-2} \quad \mathfrak{i} \in S(\Gamma, \rho, 2) .
$$

For every natural number $n$, let $T(n)$ be the sum of $\Gamma \alpha \Gamma$ such that $N(\alpha)=n$. For a prime number $p$ not dividing $d(\Phi)$, let $T\left(p^{\lambda}, p^{\mu}\right)$ denote the operator $\Gamma \alpha \Gamma$ whose elementary divisor is $\left(p^{\lambda}, p^{\mu}\right)$. Correspondingly, for every natural number $n$ prime to $d(\Phi) N$, and for a prime number $p$ not dividing $d(\Phi) N$, let $T\left(p^{\lambda}, p^{\mu} ; \mathfrak{a}\right)$ and $T(n ; \mathfrak{a})$, respectively, the operator $\Gamma_{N} \alpha \Gamma_{N}$ such that the elementary divisor of $\alpha$ is $\left(p^{\lambda}, p^{\prime \prime}\right)$ and the sum of the operators $\Gamma_{N} \alpha \Gamma_{N}$ such that $N(\alpha)=n$. We are now going to consider $\Gamma \alpha \Gamma, T(n)$ and $T(p, p)$ as the operators on $S(\Gamma, \rho, 2)$, and $\Gamma_{N} \alpha \Gamma_{N}, T(n ; \mathfrak{a})$ and $T(p, p ; \mathfrak{a})$ as the operators on $S\left(\Gamma_{N}, 2\right)$. Then our purpose of this section is to obtain the representation of $T(p ; \mathfrak{a})$ and of $T(p, p ; \mathfrak{a})$ on the representation space of $\rho_{1} \dot{+} \cdots+\rho_{r}$, making. use of that of $T(p)$ and of $T(p, p)$, respectively.

First we note that if $\Gamma_{N} \alpha \Gamma_{N}=\bigcup_{i=1}^{m} \Gamma_{N} \alpha\left(\alpha \in \Delta_{N}\right)$ is a disjoint expression, then $\Gamma \alpha \Gamma=\bigcup_{i=1}^{m} \Gamma \alpha_{i}$ is also a disjoint sum; furthermore we can choose $\alpha_{i}$ such that $\alpha_{i} \equiv\left(\begin{array}{ll}1 & 0 \\ 0 & q\end{array}\right) \bmod N$ with $q=N(\alpha)$. When $\alpha$ runs over all the elements in $\Delta_{N}$ such that 


$$
\alpha \equiv\left(\begin{array}{ll}
1 & 0 \\
0 & q
\end{array}\right) \bmod N
$$

the set of all residue classes $\alpha \bmod N$ constitutes itself an abelian group. Therefore, for suitably chosen characters $\varepsilon_{1}, \cdots, \varepsilon_{u}(u=\operatorname{deg} \rho)$ of the group $(\boldsymbol{Z} / N \boldsymbol{Z})^{*}$ we may take the representation $\rho$ of $\Omega$ such that

$$
\rho\left(\left(\begin{array}{cc}
1 & 0 \\
0 & m
\end{array}\right)\right)=\left(\delta_{\mu \nu} \varepsilon_{\nu}(m)\right) \quad(m, N)=1, \quad(1 \leqq \nu, \mu \leqq u) .
$$

On the other hand, as we can choose the representatives of $\Omega / G$ among the elements of the form (6), we have, in view of (2)

$$
\mathfrak{f} \mid(\Gamma \alpha \Gamma)=q^{-1}\left(\begin{array}{c}
\varepsilon_{1}(q)^{-1} f_{1} \mid\left(\Gamma_{N} \alpha \Gamma_{N}\right) \\
\vdots \\
\varepsilon_{u}\left(q^{-1}\right) f_{u} \mid\left(\Gamma_{N} \alpha \Gamma_{N}\right)
\end{array}\right) \quad \text { for } \quad \mathfrak{i}=\left(\begin{array}{c}
f_{1} \\
\vdots \\
f_{u}
\end{array}\right) \in S(\Gamma, \rho, 2) .
$$

Let $\left\{\mathfrak{f}_{1}, \cdots, \mathfrak{f}_{s}\right\}$ be a basis of $S(\Gamma, \rho, 2)$, on which every operator $\Gamma \alpha \Gamma$ $\left(\alpha \in \Delta_{N}\right)$ can be represented by diagonal matrix. Such a basis always exists by virtue of Proposition 3.2 in [8]. Hence for every prime number $p$ not dividing $d(\Phi) N$ we can find complex numbers $t_{\lambda}(p)$ and $t_{\lambda}(p, p)$ such that

$$
\begin{aligned}
& \mathfrak{f}_{\nu} \mid T(p)=t_{\nu}(p) \mathfrak{f}_{\nu} \\
& \mathfrak{f}_{\nu} \mid T(p, p)=t_{\nu}(p, p) \tilde{\mathfrak{f}}_{\nu} \quad(1 \leqq \nu \leqq s) .
\end{aligned}
$$

Using (8) and (9), for every component $f_{k}^{(\nu)}$ of $\mathfrak{f}_{\nu}$ we have

$$
f_{k}^{(\nu)} \mid T(p ; \mathfrak{a})=p \varepsilon_{k}(p) t_{\nu}(p) f_{k}^{(\nu)} \quad(1 \leqq \nu \leqq s, 1 \leqq k \leqq u) .
$$

Let $\gamma$ be an element in $\Gamma$ such that $\gamma \equiv\left(\begin{array}{cc}p^{-1} & 0 \\ 0 & p\end{array}\right) \bmod N$. It can be easily seen from definitions

$$
\begin{aligned}
f \mid\left(\Gamma_{N} p r \Gamma_{N}\right) & =f(r[z]) j(\gamma, z)^{2} \quad \text { for all } f \in S\left(\Gamma_{N}, 2\right), \\
T(p, p) & =\Gamma p \gamma \Gamma .
\end{aligned}
$$

As $p r \equiv\left(\begin{array}{ll}1 & 0 \\ 0 & p^{2}\end{array}\right) \bmod N$, we have by (8)

$$
\mathfrak{f} \mid T(p, p)=p^{-2}\left(\begin{array}{c}
\varepsilon_{1}(p)^{-2} f_{1} \mid T(p, p ; \mathfrak{a}) \\
\vdots \\
\varepsilon_{u}(p)^{-2} f_{u} \mid T(p, p ; \mathfrak{a})
\end{array}\right), \quad \mathfrak{f}=\left(\begin{array}{c}
f_{1} \\
\vdots \\
f_{u}
\end{array}\right) \in S(\Gamma, \rho, 2) .
$$

Consequently, for every component $f_{k}^{(\nu)}$ of $\mathfrak{f}_{\nu}$ we obtain

$$
f_{k}^{(\nu)} \mid T(p, p ; \mathfrak{a})=p^{2} \varepsilon_{k}(p)^{2} t_{\nu}(p, p) f_{k}^{(\nu)} \quad(1 \leqq \nu \leqq s, 1 \leqq k \leqq u) .
$$

Now consider $S\left(\Gamma_{N}, 2\right)$ as representation space of $\Gamma$ and decompose it into irreducible subspaces. Let $\mathfrak{r}$ be the sum of all irreducible subspaces which are equivalent to the representation space of $\rho_{i}$ for some $i$. Then we can choose a basis of $\mathfrak{r}$ among the components $f_{k}^{(\nu)}$ of $\mathfrak{f}_{\nu}$. Put $\operatorname{deg} \rho_{1}=m$. Then $u=\kappa m r$. Let $\left\{g_{1}, \cdots, g_{v}\right\}$ be a basis of $\mathfrak{r}$ obtained in such a manner. Then, for each $\mu, g_{\mu}$ is equal to $f_{k}^{(\nu)}$ for some $\nu$ and $k$. Now let $g$ be the column 
vector with components $g_{1}, \cdots, g_{v}$. Then the representation of $T(p ; \mathfrak{a})$ and $T(p, p ; a)$ with respect to $g$ are given as follows

$$
\mathfrak{g} \mid T(p ; \mathfrak{a})=\left(\begin{array}{ccc}
p \varepsilon_{i_{1}}(p) t_{\nu_{1}}(p) & 0 \\
0 & \cdot p \varepsilon_{i_{v}}(p) t_{\nu_{v}}(p)
\end{array}\right) \mathfrak{g}
$$

and

$$
\mathfrak{g} \mid T(p, p ; \mathfrak{a})=\left(\begin{array}{cc}
p^{2} \varepsilon_{i_{1}}(p)^{2} t_{\nu_{1}}(p, p) & 0 \\
0 & \dot{p}^{2} \varepsilon_{i_{v}}(p)^{2} t_{\nu_{v}}(p, p)
\end{array}\right) \mathfrak{g}
$$

where $i_{\mu}, \nu_{\mu}$ are determined by the relation $g_{\mu}=f_{k_{\mu}}^{(\nu \mu)}(1 \leqq \mu \leqq v)$.

\section{$\S 3$. Artin's $L$-functions.}

Let $F$ be a cyclotomic field containing the values of all simple characters of the group $G$. Every element $\gamma$ of $G$ defines a birational transformation of $\mathfrak{L}_{N}$, which gives rise to an element $\xi_{r}$ of $\mathcal{A}\left(J_{N}\right)$. Now we have to define an $l$-adic representation $\mathfrak{M}_{l}$ and an analytic representation $\mathfrak{M}^{d}$ for the elements in $\mathcal{A}\left(J_{N}\right) \otimes_{Q} F$. Let $l$ be a prime number which decomposes completely in $F$; we can consider $F$ as subfield of $\boldsymbol{Q}_{l}$. Let $M_{l}$ be an $l$-adic representation of $\mathcal{A}\left(J_{N}\right)$. We can extend $M_{l}$ to a representation of $\mathcal{A}_{0}\left(J_{N}\right) \otimes \boldsymbol{Q}_{l}$ in a natural manner, which we denote by $\mathfrak{M}_{l}$. Let $M^{d}$ be an analytic representation of $\mathcal{A}\left(J_{N}\right)$. Every element $\alpha$ in $\mathcal{A}\left(J_{N}\right) \otimes_{Q} F$ can be written as;

$$
\alpha=\sum_{i} a_{i} \beta_{i} \quad a_{i} \in F, \quad \beta_{i} \in \mathscr{A}\left(J_{N}\right) .
$$

Put then $\mathfrak{M}^{d}(\alpha)=\sum_{i} a_{i} M^{d}\left(\beta_{i}\right)$. Cleary, $\mathfrak{M}^{d}$ is a representation of $\mathcal{A}\left(J_{N}\right) \otimes F$.

By Theorem 2 of [7], the galois group of $K_{N} / K_{1}\left(\zeta_{N}\right)\left(K_{N}=K_{\mathfrak{a}}\right)$ is isomorphic to $G=\Gamma / \Gamma_{N}$. Put $k=\boldsymbol{Q}\left(\zeta_{N}\right)$. Let $\mathfrak{p}$ be a prime ideal of $k$ such that $\mathfrak{R}_{N}$ has no defect. Let $u$ be the generic point of $\mathfrak{L}_{N}$ such that $\boldsymbol{Q}(u)=L_{N}$, and $\tilde{u}$ be a generic point of $\mathfrak{p}\left(\mathfrak{Q}_{N}\right)$ over $k \bmod \mathfrak{p}$. Then the specialization $u \rightarrow \tilde{u}$ ref. $\mathfrak{p}$ defines a place of $K_{N}=k(u)$. For almost all $\mathfrak{p}, \mathfrak{P}\left(K_{N}\right)$ is a galois extension of $\mathfrak{P}\left(K_{1}\left(\zeta_{N}\right)\right)$ whose galois group is (canonically) isomorphic to $G$. For such a prime ideal $\mathfrak{p}$ and for any simple character $\chi$ of $G$, the local Artin's $L$-function $L(\chi, u ; \mathfrak{p})$ can be defined (cf. [9]). We recall here some formulas, concerning this function $L(\chi, u ; \mathfrak{p})$, which will be needed in the following discussions (cf. $[4,9]$ ).

The reduction mod $\mathfrak{p}$ defines an isomorphism of $\mathcal{A}_{0}\left(J_{N}\right) \otimes F$ into $\mathcal{A}_{0}\left(\tilde{J}_{N}\right) \otimes F$; denote by $\tilde{\lambda}$ the image of $\lambda$ by this isomorphism for any $\lambda$ in $\mathcal{A}_{0}\left(J_{N}\right) \otimes F$. We can choose an $l$-adic representation $\tilde{M}_{l}$ of $\mathcal{A}_{0}\left(\tilde{J}_{N}\right)$ in such a way that $\tilde{M}_{l}(\lambda)$ $=M_{l}(\tilde{\lambda})$ for $\lambda \in \mathscr{A}\left(J_{N}\right)$. We extend this representation $\tilde{M}_{l}$ to a representation $\tilde{\mathfrak{M}}_{l}$ of $\mathcal{A}_{0}\left(\tilde{J}_{N}\right) \otimes \boldsymbol{Q}_{l}$ in a natural manner. Then we have $\widetilde{\mathfrak{M}}_{l}(\lambda)=\mathfrak{M}_{l}(\tilde{\lambda})$ for every $\lambda \in \mathcal{A}_{0}\left(J_{N}\right) \otimes \boldsymbol{Q}_{l}$; so we denote hereafter $\tilde{M}_{l}$ and $\tilde{\mathfrak{M}}_{l}$ by $M_{l}$ and $\mathfrak{M}_{l}$, respectively. Now we have 


$$
L(\chi, u ; \mathfrak{p})^{m}=\operatorname{det}\left(1-\mathfrak{M}_{l}\left(\tilde{\varepsilon}_{\chi} \cdot \pi_{\mathfrak{p}}\right) u\right),
$$

where $m=\chi(1), \pi_{\mathfrak{p}}$ is the $N(\mathfrak{p})$-th power endomorphism of $J_{N}$, and $\varepsilon_{\chi}=\frac{m}{g} \sum_{\alpha \in G} \chi\left(\alpha^{-1}\right) \xi_{\alpha}$ with $g=[G: e]$.

Let $\chi_{1}, \cdots, \chi_{r}$ be distinct simple characters of $G$ with $\chi_{1}(1)=\cdots=\chi_{r}(1)=m$; then the elements $\varepsilon_{\chi_{i}}=\frac{m}{g} \sum_{\alpha \in G} \chi_{i}\left(\alpha^{-1}\right) \xi_{\alpha}(1 \leqq i \leqq r)$ are orthogonal idempotents. If we put $\chi=\chi_{1}+\cdots+\chi_{r}$ and $\varepsilon=\varepsilon_{\chi_{1}}+\cdots+\varepsilon_{\chi_{r}}$, we get

$$
\prod_{i=1}^{r} L\left(\chi_{i}, u ; \mathfrak{p}\right)^{m}=L(\chi, u ; \mathfrak{p})^{m}=\operatorname{det}\left(1-\mathfrak{M}_{l}\left(\tilde{\varepsilon} \pi_{\mathfrak{p}}\right) u\right) .
$$

DEFINITION. Let $\chi$ be a character of the group $G$. We define the global Artin's $L$-function by

$$
L\left(\chi, s ; K_{N} / K_{1}\left(\zeta_{N}\right)\right)=\prod_{\mathfrak{p}} L\left(\chi, N(\mathfrak{p})^{-s} ; \mathfrak{p}\right),
$$

where the product $\prod_{\mathfrak{p}}$ runs over all prime ideals $\mathfrak{p}$ in $k$ with a good property discribed above.

Let $\rho$ and $\rho_{1}, \cdots, \rho_{r}$ be the same as in the previous section. In the following we shall consider the Artin's $L$-functions with characters $\chi=\chi_{1}+\cdots+\chi_{r}$, $\chi_{i}=\operatorname{tr} \rho_{i}(1 \leqq i \leqq r)$. We also put

$$
\varepsilon_{\chi_{i}}=\frac{m}{g} \sum_{\alpha \in G} \chi_{i}\left(\alpha^{-1}\right) \xi_{\alpha} \quad \text { and } \quad \varepsilon=\varepsilon_{\chi_{1}}+\cdots+\varepsilon_{\chi_{r}} \quad\left(m=\operatorname{deg} \rho_{i}\right) .
$$

LEMMA 2. Let $\varphi_{n}$ be an automorphism of the field $k=\boldsymbol{Q}\left(\zeta_{N}\right)\left(\zeta_{N}=e^{\frac{2 \pi i}{N}}\right)$, such that $\zeta_{N}^{\varphi_{n}}=\zeta_{N}^{n}((n, N)=1)$. Then for every $r \in G$, the locus of $\left(u \times u^{\gamma}\right)^{\varphi_{n}}$ over $\boldsymbol{Q}$ is the locus of $u \times u^{\sigma_{n}^{-1} r \sigma_{n}}$ over $\boldsymbol{Q}$, and hence

$$
\xi_{r}^{\varphi_{n}}=\xi_{\sigma_{n}^{-1} \gamma \sigma_{n}}
$$

where $\sigma_{n}$ denotes an element in $\mathrm{o}$ such that $\sigma_{n} \equiv\left(\begin{array}{ll}1 & 0 \\ 0 & n\end{array}\right) \bmod N$.

As the proof of this Lemma is the same as in [4], we omit it.

Let $\mathcal{A}\left(J_{N} ; k\right)$ and $\mathscr{A}\left(J_{N} ; \boldsymbol{Q}\right)$ be, respectively, the sets of elements in $\mathscr{A}\left(J_{N}\right)$. which are defined over $k$ and over $\boldsymbol{Q}$. The automorphism $\varphi_{n}$ of $k=\boldsymbol{Q}\left(\zeta_{N}\right)$ gives an automorphism of $\mathcal{A}\left(J_{N} ; k\right)$; this can be extended to an automorphism. of $\mathcal{A}_{0}\left(J_{N} ; k\right) \otimes F$ by putting

$$
\left(\sum_{i} a_{i} \lambda_{i}\right)^{\varphi_{n}}=\sum_{i} a_{i} \lambda_{i}^{\varphi_{n}}
$$

for $a_{i} \in F, \lambda \in \mathcal{A}\left(J_{N} ; k\right)$. Then, by (18), for every element $\alpha=\sum \alpha_{i} \xi_{\gamma_{i}}$ with $a_{i} \in F, \gamma_{i} \in G$, we have

$$
\alpha^{\varphi}=\sum_{i} \alpha_{i} \xi_{\sigma_{n}^{-1} \gamma_{i} \sigma_{n}}
$$

By definition of $\varepsilon$, Proposition 1 in $\S 2$ and Lemma 2 we get 


$$
\begin{gathered}
\varepsilon^{\varphi_{n}}=\varepsilon \quad(n, N)=1, \\
\varepsilon \cdot \xi_{p}=\xi_{p} \cdot \varepsilon \quad \text { and } \quad \varepsilon \cdot \eta_{p}=\eta_{p} \cdot \varepsilon, \\
\zeta \cdot \varepsilon=\varepsilon \cdot \zeta .
\end{gathered}
$$

Furthermore, by the same argument as in [4] we can easily prove

$$
\widetilde{\varepsilon} \cdot \pi_{p}=\pi_{p} \cdot \widetilde{\varepsilon} .
$$

It is easy to see that $\mathcal{A}\left(J_{N} ; \boldsymbol{Q}\right) \otimes F$ is the set of all elements $\alpha$ of $\mathcal{A}\left(J_{N} ; k\right) \otimes F$ such that $\alpha^{\varphi_{n}}=\alpha$ for every $n$ prime to $N$. By our definition of $\mathfrak{M}_{l}$ and $\mathfrak{M}^{d}$, if we restrict them to $\mathcal{A}\left(J_{N} ; \boldsymbol{Q}\right) \otimes F, \mathfrak{M}_{l}$ is equivalent to the representation $\left(\begin{array}{cc}\mathfrak{M}^{d} & 0 \\ 0 & \mathfrak{M}^{d}\end{array}\right)^{1)}$. As $\xi_{p}$ and $\eta_{p}$ are defined over $\boldsymbol{Q}$, we have $\left(\xi_{p} \cdot \varepsilon\right)^{\varphi_{n}}=\xi_{p} \cdot \varepsilon,\left(\eta_{p} \cdot \varepsilon\right)^{\varphi_{n}}$ $=\eta_{p} \cdot \varepsilon$, for every $n$ prime to $N$. Hence we obtain

$$
\begin{aligned}
& \mathfrak{M}_{l}\left(\widetilde{\varepsilon} \cdot \tilde{\xi}_{p}\right)=\mathfrak{M}_{l}\left(\varepsilon \cdot \xi_{p}\right) \sim\left(\begin{array}{cc}
\mathfrak{M}^{d}\left(\varepsilon \cdot \xi_{p}\right) & 0 \\
0 & \mathfrak{M}^{d}\left(\varepsilon \cdot \xi_{p}\right)
\end{array}\right) \\
& \mathfrak{M}_{l}\left(\widetilde{\varepsilon} \cdot \tilde{\eta}_{p}\right)=\mathfrak{M}_{l}\left(\varepsilon \cdot \eta_{p}\right) \sim\left(\begin{array}{cc}
\mathfrak{M}^{d}\left(\varepsilon \cdot \eta_{p}\right) & 0 \\
0 & \mathfrak{M}^{d}\left(\varepsilon \cdot \eta_{p}\right)
\end{array}\right) .
\end{aligned}
$$

Using the formulas (20)-(25) and congruence relations (1), we get

$$
\operatorname{det}\left(1-\mathfrak{M}_{l}\left(\widetilde{\varepsilon} \cdot \pi_{p}\right) X\right)=\operatorname{det}\left(1-\mathfrak{M}^{d}\left(\varepsilon \cdot \xi_{p}\right) X+p^{d} \mathfrak{M}^{d}\left(\varepsilon \cdot \eta_{p}\right) X^{2}\right) .
$$

Let $\psi(n)$ be a character of the multiplicative group $(\boldsymbol{Z} / N \boldsymbol{Z})^{*}$ of integers $n \bmod N$ which are prime to $N$ such that $\psi(n)=a$ primitive $f_{n}$-th root of unity, where $f_{n}=$ the smallest positive integer satisfying $n^{f_{n}} \equiv 1 \bmod N$. If $p$ does not divide $N, p$ decomposes completely in $k$, i. e., we have $p=\mathfrak{p}_{1} \cdots \mathfrak{p}_{g_{p}}$ and $N\left(\mathfrak{p}_{i}\right)=p^{f_{p}}\left(1 \leqq i \leqq g_{p}\right)$ with $f_{p} \cdot g_{p}=\varphi(N)$. Therefore we obtain

$$
\begin{aligned}
\prod_{p \nmid N} \operatorname{det} & \left(1-\mathfrak{M}_{l}\left(\tilde{\varepsilon} \pi_{p}\right) N(\mathfrak{p})^{-s}\right) \\
& =\prod_{p \nmid N} \prod_{i=1}^{g_{p}} \operatorname{det}\left(1-\mathfrak{M}_{l}\left(\widetilde{\varepsilon} \pi_{\mathfrak{p}_{i}}\right) N\left(\mathfrak{p}_{i}\right)^{-s}\right) \\
& =\prod_{p \nmid N} \prod_{i=1}^{g_{p}} \prod_{\mu=1}^{f_{p}} \operatorname{det}\left(1-\mathfrak{M}_{l}\left(\widetilde{\varepsilon} \pi_{p}\right) \psi(p)^{\mu} p^{-s}\right) \\
& =\prod_{p \nmid N} \prod_{i=1}^{\varphi(N)} \operatorname{det}\left(1-\mathfrak{M}_{l}\left(\widetilde{\varepsilon} \pi_{p}\right) \psi(p)^{\mu} p^{-s}\right) .
\end{aligned}
$$

Then by virtue of (16), (17), (26), we have

$$
\begin{aligned}
L\left(\chi, s ; K_{N} / K_{1}\left(\zeta_{N}\right)\right)^{m}= & \prod_{\mathfrak{p}} \operatorname{det}\left(1-\mathfrak{M}_{l}\left(\widetilde{\varepsilon} \cdot \pi_{\mathfrak{p}}\right) N(\mathfrak{p})^{-s}\right) \\
= & \prod_{p}^{\varphi} \prod_{\mu=1}^{\varphi(N)} \operatorname{det}\left(1-\mathfrak{M}_{l}\left(\widetilde{\varepsilon} \cdot \pi_{p}\right) \psi(p)^{\mu} p^{-s}\right) \\
= & \prod_{p}^{\varphi(N)} \prod_{\mu=1}^{(N)} \operatorname{det}\left(1-\mathfrak{M}^{d}\left(\varepsilon \cdot \xi_{p}\right) \psi(p)^{\mu} p^{-s}\right. \\
& \left.+\mathfrak{M}^{d}\left(\varepsilon \cdot \eta_{p}\right) \psi(p)^{2 \mu} p^{1-2 s}\right),
\end{aligned}
$$

1) We can take $M^{d}$ in such a way that $M^{d}(\alpha)$ is of rational coefficients for $\alpha$ $\in \mathcal{A}\left(J_{N} ; \boldsymbol{Q}\right)$, then $\mathfrak{M}^{d}(\alpha)$ has coefficients in $F$ for every $\alpha \in \mathcal{A}\left(J_{N} ; \boldsymbol{Q}\right) \otimes F$. 
where $p$ runs over almost all primes in $\boldsymbol{Q}$ not dividing $N$.

As the representations $\mathfrak{M}^{d}\left(\xi_{p}\right)$ and $\mathfrak{M}^{d}\left(\eta_{p}\right)$ are, respectively, equal to the representations of the operators $T(p ; \mathfrak{a})$ and $T(p, p ; \mathfrak{a})$ with respect to a certain basis of $S\left(\Gamma_{N}, 2\right)$, the representations $\mathfrak{M}^{d}\left(\varepsilon \cdot \xi_{p}\right)$ and $\mathfrak{M}^{d}\left(\varepsilon \cdot \eta_{p}\right)$ are, respectively, equivalent to the representations of $T(p ; \mathfrak{a})$ and $T(p, p ; \mathfrak{a})$ on $\mathfrak{r}$ given in (13) and (14)] For if we consider $\varepsilon$ as an operator on $S\left(\Gamma_{N}, 2\right), \varepsilon$ vanishes outside $\mathfrak{r}$ and is the identity on $\mathfrak{r}$.

Substituting the expressions (13) and (14), respectively, for $\mathfrak{M}^{d}\left(\varepsilon \cdot \xi_{p}\right)$ and $\mathfrak{M}^{d}\left(\varepsilon \cdot \eta_{p}\right)$, we have

$$
\begin{aligned}
L\left(\chi, s ; K_{N} / K_{1}\left(\zeta_{N}\right)\right)^{m}= & \prod_{\mu=1}^{\varphi(N)} \prod_{k=1}^{v}\left\{\prod _ { p } \left(1-\varepsilon_{i_{k}}(p) \psi(p)^{\mu} t_{\nu_{k}}(p) p^{1-s}\right.\right. \\
& \left.\left.+\varepsilon_{i_{k}}(p)^{2} \psi(p)^{2 \mu} t_{\nu_{k}}(p, p) p^{3-2 s}\right)\right\} .
\end{aligned}
$$

For every natural number $n$ prime to $N$, let $\mathfrak{I}(n)$ be the representation of $T(n)$ with respect to the basis $\left\{\mathfrak{f}_{1}, \cdots, \mathfrak{f}_{s}\right\}$ (considered in $\S 2$ ). For every prime number $p \nmid d(\Phi) N$, let $\mathcal{I}(p, p)$ be the representation of $T(p, p)$ with respect to the above basis. Put

$$
\mathfrak{D}\left(s ; \varepsilon_{i_{k}} \psi^{\prime \prime}\right)=\sum_{(n, N)=1} \mathfrak{I}(n) \varepsilon_{i_{k}}(n) \psi(n)^{\prime \prime} n^{-s} \quad(1 \leqq \mu \leqq \varphi(N), 1 \leqq k \leqq v) .
$$

This is just a special case of $\mathscr{D}(s ; \xi)$ in $[8$, Theorem 1$]$.

By our choice of the basis $\left\{\mathfrak{f}_{1}, \cdots, \mathfrak{f}_{s}\right\}, \mathfrak{I}(n)$ and $\mathfrak{I}(p, p)$ are diagonal and diagonal elements are $t_{\lambda}(n)$ and $t_{\lambda}(p, p)$, respectively. Therefore the $\nu_{k}$-th diagonal element of $\mathscr{D}\left(s ; \varepsilon_{i_{k}} \psi^{\prime \prime}\right)$ is written as;

$$
\begin{aligned}
D_{\nu_{k}}\left(s ; \varepsilon_{i_{k}} \psi^{\mu}\right)= & \sum_{(n, N)=1} t_{\nu_{k}}(n) \varepsilon_{i_{k}}(n) \psi(n)^{\mu} n^{-s} \\
= & \prod_{\substack{p l d(d) \\
p \nmid N}}\left(1-t_{\nu_{k}}(p) \varepsilon_{i_{k}}(p) \psi(p)^{\mu} p^{-s}\right)^{-1} \\
& \times \prod_{p \nmid d(\Phi) N}\left(1-t_{\nu_{k}}(p) \varepsilon_{i_{k}}(p) \psi(p)^{\mu} p^{-s}\right. \\
& \left.+t_{\nu_{k}}(p, p) \varepsilon_{i_{k}}(p)^{2} \psi(p)^{2 \mu} p^{1-2 s}\right)^{-1} .
\end{aligned}
$$

Hence, from (28) we have

$$
L\left(\chi, s ; K_{N} / K_{1}\left(\zeta_{N}\right)\right)^{m}=f(s) \times \prod_{\mu=1}^{\varphi(N)} \prod_{k=1}^{v} D_{\nu_{k}}\left(s-1 ; \varepsilon_{i_{k}} \psi^{\mu}\right)^{-1},
$$

where $f(s)$ denotes a rational function in finite number of $p^{-s}$. Consequently by Theorem 1 of [8], we have the following theorem:

THEOREM 1. Let $\rho_{1}$ be an absolutely irreducible representation of $G$ of degree $m$ which is contained in the analytic representation of $G$ on the jacobian variety $J_{N}$. Considering $G$ as a normal subgroup of $\Omega=G L(2, \boldsymbol{Z} / N \boldsymbol{Z}) /\{ \pm 1\}$, let $\rho$ be the irreducible representation of $\Omega$ which is an extension of $\rho_{1}$. Let $\rho$ be equivalent to $\kappa\left(\rho_{1} \dot{+} \cdots \dot{+} \rho_{r}\right)$ on $G$ with multiplicity $\kappa$ and inequivalent representations $\rho_{1}, \cdots, \rho_{r}$ of $G$ which are conjugates of $\rho_{1}$ relative to $\Omega$. Put $\chi_{i}=\operatorname{tr} \rho_{i}$, 
$\chi=\chi_{1}+\cdots+\chi_{r}$. Then the $m$-th power of Artin's L-function $L\left(\chi, s ; K_{N} / K_{1}\left(\zeta_{N}\right)\right)^{m}$ can be expressed in the form

$$
L\left(\chi, s ; K_{N} / K_{1}\left(\zeta_{N}\right)\right)^{m}=f(s) \times \prod_{\mu=1}^{\varphi(N)} \prod_{k=1}^{v} D_{\nu_{k}}\left(s-1 ; \varepsilon_{i_{k}} \psi^{(\mu)}\right)^{-1},
$$

where $f(s)$ is a rational function of $p^{-s}$ and the $D_{\nu_{k}}\left(s ; \varepsilon_{i_{k}} \psi^{\prime \prime}\right)$ are Dirichlet series with characters $\varepsilon_{i_{k}} \psi^{\prime \prime}$ obtained from the representation of modular correspondences by automorphic cusp forms of type $(\Gamma, \rho, 2)$.

Therefore $L\left(\chi, s ; K_{N} / K_{1}\left(\zeta_{N}\right)\right)^{m}$ can be continued meromorphically to the whole $s$-plane and satisfies a functional equation.

It is desirable to show that the Artin's $L$-function itself satisfies the properties in Theorem 1. We now prove that this is in fact true when $\rho \sim \rho_{1}$, i. e., $r=\kappa=1$ in (4).

Let $\mathfrak{f}_{1}, \cdots, \mathfrak{f}_{s}$ be the basis of $S(\Gamma, \rho, 2)$. As the representation $\rho_{1}$ of $G$ is irreducible, the set of all components $f_{k}^{(\nu)}$ of $\mathfrak{f}_{\nu}\left(1 \leqq \nu \leqq s, 1 \leqq k \leqq m, m=\operatorname{deg} \rho_{1}\right)$ constitutes a basis for the space $\mathfrak{r}$ defined in $\S 2$. By (8), the representation of $T(p ; \mathfrak{a})$ with respect to $\left\{f_{k}^{(1)}, \cdots, f_{k}^{(s)}\right\}$ is $p \varepsilon_{k}(p) \mathfrak{I}(p)(1 \leqq k \leqq m)$. We put ${ }^{t} \mathfrak{g}_{1}$ $=\left(f_{1}^{(1)}, \cdots, f_{1}^{(s)}, \cdots, f_{m}^{(1)}, \cdots, f_{m}^{(s)}\right)$. Then the representation of $T(p ; \mathfrak{a})$ with respect to $g_{1}$ is given by

$$
\mathfrak{g}_{1} \mid T(p ; \mathfrak{a})=\left(\begin{array}{cc}
p \varepsilon_{1}(p) \mathfrak{I}(p) & 0 \\
0 & \dot{p} \varepsilon_{m}(p) \mathfrak{I}(p)
\end{array}\right) g_{1} \text { for } p \times N .
$$

Similarly, by (11), we obtain the representation of $T(p, p ; \mathfrak{a})$ as

$$
g_{1} \mid T(p, p ; \mathfrak{a})=\left(\begin{array}{cc}
p^{2} \varepsilon_{1}(p)^{2} \mathfrak{I}(p, p) & 0 \\
0 & \dot{p}^{2} \varepsilon_{m}(p)^{2 \mathfrak{T}(}(p, p)
\end{array}\right) g_{1} \quad \text { for } p \times d(\Phi) N .
$$

Substituting these expressions for $\mathfrak{M}^{d}\left(\varepsilon \cdot \xi_{p}\right)$ and $\mathfrak{M}^{d}\left(\varepsilon \cdot \eta_{p}\right)$, respectively, we have by (27)

$$
\begin{aligned}
L\left(\chi, s ; K_{N} / K_{1}\left(\zeta_{N}\right)\right)^{m}= & \prod_{\mu=1}^{\varphi(N)} \prod_{k=1}^{m}\left\{\prod _ { p } \operatorname { d e t } \left(1-\mathfrak{T}(p) \varepsilon_{k}(p) \psi(p)^{\mu} p^{1-s}\right.\right. \\
& \left.\left.+\mathfrak{T}(p, p) \varepsilon_{k}(p)^{2} \psi(p)^{2 \mu} p^{3-2 s}\right)\right\} .
\end{aligned}
$$

As the $\varepsilon_{i}$ are characters of $(\boldsymbol{Z} / N \boldsymbol{Z})^{*}(1 \leqq i \leqq m)$, if $p=p_{1} \cdots p_{g_{p}}$ in $k=\boldsymbol{Q}\left(\zeta_{N}\right)$ with $N\left(\mathfrak{p}_{i}\right)=p^{f_{p}}\left(1 \leqq i \leqq \mathfrak{p}_{p}\right)$, then $p^{f_{p}} \equiv 1 \bmod N$, and the $\varepsilon_{i}(p)$ are $f_{p}$-th. roots of unity $(1 \leqq i \leqq m)$, moreover, $\psi(p)$ is a primitive $f_{p}$-th root of unity. Thus we obtain

$$
L\left(\chi, s ; K_{N} / K_{1}\left(\zeta_{N}\right)\right)=\prod_{\mu=1}^{\varphi(N)}\left\{\prod_{p} \operatorname{det}\left(1-\mathfrak{I}(p) \psi(p)^{\mu} p^{1-s}+\mathfrak{I}(p, p) \psi(p)^{2 \mu} p^{3-2 s}\right)\right\} .
$$

Now put

$$
\mathfrak{D}\left(s ; \psi^{\prime \prime}\right)=\sum_{(n, N)=1} \mathfrak{I}(n) \psi(n) n^{-s} \quad(1 \leqq \mu \leqq \varphi(N)) .
$$

By Theorem 1 of [8], (29) can be written as ; 


$$
\begin{aligned}
\mathfrak{D}\left(s ; \psi^{\prime \prime}\right) & =\prod_{\substack{p|d|(\boldsymbol{D}) \\
p \nmid N}}\left(1-\mathfrak{I}(p) \psi(p)^{\mu} p^{-s}\right)^{-1} \\
& \times \prod_{p \nmid d(\Phi) N}\left(1-\mathfrak{I}(p) \psi(p)^{\mu} p^{-s}+\mathfrak{I}(p, p) \psi(p)^{2 \mu} p^{1-2 s}\right)^{-1}
\end{aligned}
$$

Thus the Artin's $L$-function can be expressed as

$$
L\left(\chi, s ; K_{N} / K_{1}\left(\zeta_{N}\right)\right)=f(s) \times \prod_{\mu=1}^{\varphi(N)} \operatorname{det} \mathfrak{D}\left(s-1, \psi^{\prime \prime}\right),
$$

where $f(s)$ denotes a rational function of $p^{-s}$.

THEOREM 2. When $\rho_{1}$ is equivalent to $\rho$ in the Theorem 1, the Artin's $L$ function can be expressed as

$$
L\left(\chi, s ; K_{N} / K_{1}\left(\zeta_{N}\right)\right)=f(s) \times \prod_{\mu=1}^{\varphi(N)} \operatorname{det} \mathfrak{D}\left(s-1, \psi^{\mu}\right)^{-1},
$$

where $f(s)$ is a rational function of $p^{-s}$ and $\mathfrak{D}\left(s, \psi^{\mu}\right)(1 \leqq \mu \leqq \varphi(N))$ are Dirichlet series with characters $\psi^{\mu}$, obtained from the representation of modular correspondences by automorphic cusp forms of type $(\Gamma, \rho, 2)$.

\section{Department of Mathematics Osaka University}

\section{References}

[1] A.H. Clifford, Representations induced in an invariant subgroups, Ann. of Math., 38 (1937), 533-550.

[2] M. Eichler, Quaternäre quadratische Formen und die Riemannsche Vermutung für die Kongruenzzetafunktion, Arch. Math., 5 (1954), 355-366.

[3] E. Hecke, Über Modulfunktionen und die Dirichletschen Reihen mit Eulerscher Produktentwicklung, I, II, Math. Ann., 114 (1937), 1-28; 316-351.

[4] S.S. Rangachari, Modulare Korrespondenzen und L-Reihen, J. Reine Angew. Math., 205 (1961), 119-155.

[5] G. Shimura, Correspondences modulaires et les fonctions $\zeta$ de courbes algebriques, J. Math. Soc. Japan, 10 (1958), 1-28.

[6] G. Shimura, On the theory of automorphic functions, Ann. of Math., 70 (1959), 101-144.

[7] G. Shimura, On the zeta-functions of the algebraic curves uniformized by certain automorphic functions, J. Math. Soc. Japan, 13 (1961), 275-331.

[8] G. Shimura, On Dirichlet series and abelian varieties attatched to automorphic forms, Ann. of Miath., 76 (1962), 237-294.

[9] A. Weil, Sur les courbes algebriques et les varietes qui s'en deduisent, Hermann, Paris, 1948.

[10] A. Weil, Varietes abeliennes et courbes algebriques, Hermann, Paris, 1948. 